\title{
Inherited Thrombocytopenia with a Different Type of Gene Mutation: A Brief Literature Review and Two Case Studies
}

\author{
Mohammad Taghi Arzanian, ${ }^{1,}$ \\ ${ }^{1}$ Pediatric Hematologist-Oncologist, Congenital Hematological Disorders Research Center, Mofid Children's Hospital, Shahid Beheshti University of Medical Sciences, Tehran, \\ IR Iran \\ "Corresponding author: Mohammad Taghi Arzanian, Pediatric Hematologist-Oncologist, Congenital Hematological Disorders Research Center, Mofid Children's Hospital, \\ Shahid Beheshti University of Medical Sciences, Tehran, IR Iran. E-mail: mtarzanian@gmail.com
}

Received 2016 January 06; Revised 2016 February 08; Accepted 2016 March 09.

\begin{abstract}
Hereditary thrombocytopenias are rare bleeding disorders, which cause a deficiency of platelets in early infancy. This group of disorders is sometimes associated with abnormal phenotypes, like absence of radius. Diagnosis of this type of thrombocytopenia is usually difficult; other causes of thrombocytopenia, such as immune disorders and infections, must be ruled out. The symptoms of hereditary thrombocytopenia also vary from seldom and mild to severe bleeding and occasionally may first occur in late childhood. In this group of patients, we must differentiate heritable disorders from the acquired types of thrombocytopenia, like immune thrombocytopenic purpura. It is also important to watch for pitfalls to avoid unnecessary and potentially hazardous treatment. Herein, we briefly review the recent literature on hereditary thrombocytopenia and then present the cases of two referred patients. The first case had suffered from persistent thrombocytopenia since early infancy and was diagnosed with congenital amegakaryocytic thrombocytopenia, while the other patient presented with Wiskott - Aldrich syndrome.
\end{abstract}

Keywords: Hereditary Thrombocytopenia, Mean Platelet Volume (MPV), Non-Muscle Myosin, NMMHC-IIA

\section{Context}

Bleeding syndromes due to inherited defects of platelet production are a heterogeneous group of platelet disorders of growing importance. Once considered rare, hereditary thrombocytopenias are currently being recognized with increasing frequency. Each presentation of hereditary thrombocytopenia is unique. They may range from severe bleeding that has an early presentation to mild conditions that may remain undetected into adulthood. In patients with a late presentation, distinguishing between inherited and acquired thrombocytopenia, especially immune thrombocytopenic purpura (ITP), is crucial to avoid unnecessary and potentially harmful treatments in patients who present later in their disease course $(1,2)$.

\section{Evidence Acquisition}

\subsection{Physiology of Platelets}

Platelets are small anucleate cells that measure 2.4 $4.0 \mu \mathrm{m}$ in size. Thrombopoiesis takes place in a pluripotent hematopoietic stem cell that undergoes "commitment" through megakaryocyte (MK) transcription factors, such as GATA1, FOG, and FLI1. The committed megakaryoblast acts as a progenitor cell when it undergoes cell division. During this process, MK burst-forming units and MK colony-forming units (CFU-MKs) give rise to either large or small MK colonies. CFU-MKs loses the capacity to divide and begins the process of endoreduplication, which leads to polyploidization and cellular enlargement. Finally, the immature MK completes its terminal differentiation into a mature MK with many polyploid nuclei, an expanded size, extensive intracellular organelles for platelet function, and membrane structures for platelet shedding (1).

Many genes are involved in this process: transcription factors, cytokines, cell surface receptor molecules for signaling, molecular motors, and regulators of the cell cycle. Any mutations in these genes may decrease the production or the lifespan of platelets. The small size of platelets allows them to flow easily through the vessels and make contact with damaged vessel walls and endothelia, where they are activated to progress through different stages, like adhesion, the release of many molecules, aggregation, exposure to procoagulant factors to form microparticles, and finally forming a clot. This clot produces a plug to occlude the damaged vessel wall and prevent bleeding (1-3). 


\subsection{Thrombocytopenia}

The etiology and symptoms of thrombocytopenia are very different. Platelet counts up to $150 \times 10^{9} / 1$ are considered normal, while those that are less than $100 \times 10^{9} / 1$ are pathologic $(1,2)$.

Platelets have a very important function in preserving vessel wall integrity and also play a primary role in hemostatic processes. A platelet count of less than $10-20 \times$ $10^{9} / 1$ may cause spontaneous bleeding. In new patients of any age, the most common cause of thrombocytopenia is acquired, such as an immune disease that includes platelet destruction or consumption, infection, drug induction, and bone marrow failure. The etiology of thrombocytopenia also varies across different ages; for example, in newborns, the most common causes are infections and immune thrombocytopenia. In older children, viral infections and immune thrombocytopenia are frequently observed $(1,4)$.

\subsection{Hereditary Thrombocytopenia}

Hereditary thrombocytopenias are rare diseases and usually appear in infancy; sometimes, they are associated with other abnormal findings. Recent molecular genetic explorations have led to a diagnosis of the etiology of most of these abnormal findings, which are assigned a different type of classification based upon the size of the platelets and their particular genetic mutation, as well as their mode of inheritance (1-3). The age at presentation and the chronicity of the diseases are also important. Severe forms of hereditary thrombocytopenia and platelet dysfunction usually appear quite early in infancy. Mild forms may be recognized during later life or even in adulthood as incidental findings following a routine complete blood count (CBC) examination (1-4). In these cases, it is always necessary to ask about a previous history of ecchymosis or bleeding diathesis, such as menorrhagia. In these cases, a careful evaluation of the patients may be useful to determine the cause, including clinical syndromes or other causes of thrombocytopenia $(1,2,4)$.

A review of the peripheral blood smear (PBS) to evaluate the size of the platelets is important because size is associated with certain conditions of thrombocytopenia. For example, in Bernard Soulier syndrome(BSS), the size of the platelets is larger than normal and the mean platelet volume (MPV) is elevated. In contrast, the platelets can be too small in Wiskott - Aldrich syndrome (WAS). Abnormal granules in the neutrophils and also in platelet-like Dohle bodies can help to establish the diagnosis. Asking about a history of thrombocytopenia and a bleeding tendency in the family is recommended.

In newborns who present with bleeding diathesis at birth, abnormal findings like absence of radius, kidney problems, hearing loss, and cataracts are important factors for the evaluation and diagnosis of specific thrombocytopenic syndromes $(1,2,4)$.

Chronic thrombocytopenia or bleeding issues that appear to be out of proportion with the platelet numbers are often important points to use to rule out immune thrombocytopenia. Bleeding dysfunctions like ecchymosis, epistaxis, bleeding of the gums, menorrhagia, and abnormal bleeding during surgical procedures and dental extractions are significant. Therefore, in all these situations, it is necessary to consider inherited thrombocytopenia $(1,5)$.

\subsection{Diagnosis}

The following tests are recommended to evaluate the causes of thrombocytopenia:

1. CBC and MPV.

2. Review of a PBS.

3. Coagulation profiles.

4. Bleeding time (BT).

5. Platelet adhesion.

6. Platelet aggregation.

7. Platelet granule content and release reactions.

8. Platelet flow cytometry.

9. Electron microscopy.

10. Genetic analysis.

11. Analysis of receptor expression, protein phosphorylation, and the formation of second messengers (2).

\subsection{Classification of Hereditary Thrombocytopenia}

For better management of hereditary thrombocytopenias, it is necessary to categorize them. Different methods have been used for this classification. The size of the platelets, age at thrombocytopenia presentation, severity of bleeding, any associated abnormal findings, and the results of a molecular analysis are used for classification (6).

\subsection{MYH9-Related Disorders: A Group of Autosomal-Dominant Diseases}

The history of this group of thrombocytopenias goes back to Richard May, who found inclusion bodies (Dohle bodies) in neutrophils and thrombocytopenia with giantsized platelets in 1945 . This disorder was confirmed by Hegglin and recognized as having an autosomal dominant mode of inheritance. Later, in 1985, another type of macro thrombocytopenia with a dominant mode of inheritance was reported (Fechtner Syndrome) that occurs along with cataracts, deafness, and nephritis (2-6). Ten years ago, it was shown that all these abnormal findings are caused by a mutation of $\mathrm{MYH9}$, the gene that encodes for the heavy chain A of non-muscle myosin of class II (NMMHC-IIA). 
The myosin family genes play an important role in cellular processes, consist of two heavy chains and two light chains, and are located on chromosome 22q. So far, 44 mutations of MYH9 have been reported $(1,3,4,6)$. These patients have mild bleeding and macrothrombocytopenia, which typically includes four different problems: May-Hegglin anomaly (MHA) and Sebastian, Epstein, and Fechtnes Syndromes. Megakaryocyte maturation defects cause the thrombocytopenia, which is usually quite severe (Table 1) (1, 2, 5-7).

The severity of bleeding in MYH9 disorders varies. Most patients report mild bleeding, which is perhaps due to their large platelets. The presentation of bleeding includes epistaxis, ecchymosis, gum bleeding, or menorrhagia. Sometimes, the patients need platelet transfusions prior to surgical procedures (2, 5-7). Renal problems may be seen in $30 \%$ of these patients, while $15 \%$ report cataract and lens problems. Hearing loss is also common and may be observed in $60 \%$ of patients (7).

The laboratory findings indicate that the size of the platelets is large, even larger than in ITP, and the number of platelets varies from $10 \times 10^{9} / 1$ to $150 \times 10^{9} / 1$. Dohle-like bodies may be seen in neutrophils in about $40 \%-80 \%$ of cases. Dohle bodies are actually aggregates of NMMHC-IIA. The bone marrow is usually normal. Platelet surface glycoprotein Ib/IX/V is occasionally lower than normal in some patients $(1,4,6,7)$.

\subsubsection{Diagnosis of MYH9}

The hallmarks of the disease are thrombocytopenia in early infancy with a large platelet size and an autosomal dominant mode of inheritance. Some patients experience deafness, cataracts, or nephritis. These patients may be misdiagnosed with ITP. When the case is suspect for MYH9, it can be confirmed by immunofluorescence testing for NMMHC-IIA. Proper evaluation of the genetic markers and specific mutations is very important. In addition, these patients must be checked for cataracts and hearing loss (1-4).

The other causes of macrothrombocytopenia include the following:

-Chronic autoimmune thrombocytopenia.

-BSS. In this disorder, the expression of glycoprotein $\mathrm{Ib} / \mathrm{IX} / \mathrm{V}$ receptors is usually reduced or totally absent. The mode of inheritance is autosomal recessive (1-4).

- Gray Syndrome: Giant platelets are found; $\alpha$ granules of platelets are empty, and moderate thrombocytopenia is present. The type of inheritance is autosomal recessive. On the $\mathrm{PBC}$, platelets appear pale and gray $(1,3,4)$.

-Paris-Trousseau Syndrome: In this disorder, platelets are large; the patients are mentally retarded with cardiac problems and facial anomaly. There is a mutation of the GATA-1 gene on XPII-12 (1).
-Familial platelet disorder with myeloid leukemia: A moderate thrombocytopenia is present; the platelet size is normal, but there is an aspirin-like function defect in platelet aggregation. These patients have a tendency to develop myeloid leukemia. The mode of inheritance of this disorder is autosomal dominant. The gene defect is located on chromosome 21q22.1-22.2.

-Mediterranean macrothrombocytopenia: The thrombocytopenia is typically mild to moderate, and there is no significant bleeding tendency. The expression of glycoprotein Ib-IX-V is diminished. This gene mutation is located on chromosome 17 (1).

-Di-George (Velo-cardiofacial) Syndrome: In this disorder, there are cardiac abnormalities, thymus and parathyroid defects, and learning disorders along with facial abnormalities. It is called CATCH22; these patients have mild thrombocytopenia, mild bleeding problems, and a deletion of chromosome 22q11. In some patients, a von Willebrand factor receptor defect (GP1BA, GP1BB, GPS, GPq) may be seen. These patients often do not require thrombocytopenia treatment (1).

-Thrombocytopenia with a defect on chromosome 10: Mild thrombocytopenia is present. The size and shape of platelets are normal, and there is no bleeding tendency. Small megakaryocytes and hypolobulated nuclei are present in the bone marrow. This genetic disorder is linked to the short arm of chromosome 10p11-12.28.29 (1, 2, 5-7).

\subsubsection{Treatment}

Patients with platelet disorders should avoid aspirin, which inhibits platelet aggregation, as well as some antihistamines and any drugs that interfere with platelet function. These patients should also be careful to avoid trauma and unnecessary or invasive dental surgery; good dental hygiene is therefore essential. Platelet transfusion may be necessary when surgery is required. Platelet counts of more than $50 \times 10^{9} / 1$ for major surgery or invasive procedures and greater than $100 \times 10^{9} / 1$ for operations at sites where coagulation is critical, including eye surgery and neurosurgery, are recommended $(1-4,6)$.

Platelet transfusion sometimes may result in alloimmunization, so the use of human leukocyte antigen (HLA)matched platelets is recommended. Oral thrombopoietin receptor agonists like Eltrombopag are prescribed for the management of bleeding, particularly in surgical procedures. Desmopressin (DDAVP) may also be used for hemostasis. Tranexamic acid and recombinant activated factor VII are also useful to manage bleeding, and bone marrow transplantation has been carried out in several cases of severe coagulation disorders $(1,2,5-8)$. 
Table 1. Clinical and Laboratory Findings in MYH9-Related Diseases

\begin{tabular}{|c|c|c|c|c|}
\hline Type of Disease & МНА & FS & EPS & SPS \\
\hline Macrothrombocytopenia & + & + & + & + \\
\hline Inclusion bodies & + & + & - & + \\
\hline Hearing loss & - & + & + & - \\
\hline Nephritis & - & + & + & - \\
\hline Cataracts & - & + & - & - \\
\hline
\end{tabular}

\subsection{Congenital Amegakaryocytic Thrombocytopenia}

Congenital amegakaryocytic thrombocytopenia (CAMT) is a rare condition with autosomal recessive inheritance. The thrombocytopenia is usually severe and causes bleeding during the first days of life, which may be mistaken for immune thrombocytopenia of the newborn. Platelet transfusion is typically required, and the diagnosis is made by bone marrow aspiration (BMA), where virtually no megakaryocytes are seen. The etiology of the disease is due to a mutation in the MPL gene caused by the altered expression or function of the TPO receptor (c-MPL). Patients with congenital amegakaryocytic thrombocytopenia present during the newborn period with severe bleeding. This type of hereditary thrombocytopenia may progress to bone marrow failure within 5 - 10 years. The reason for bone marrow failure is not completely understood, but it appears that TPO and c-MPL are necessary for the growth and maintenance of the hematopoietic stem cell population. CAMT must be differentiated from TAR syndrome and WAS. There is usually no physical anomaly in CAMT; in contrast, patients with WAS have small platelets, skin problems, and immune (B-T cell) defects. CAMT should also be distinguished from any type of aplastic anemia, like Fanconi's anemia. A molecular analysis of the MPL gene is used for the definitive diagnosis; CAMT is treated with hematopoietic stem cell transplantation (HSCT) $(2,9$, 10).

\subsection{X-Linked Macrothrombocytopenia}

This type of thrombocytopenia is usually associated with erythrocyte problems, dyserythropoesis, and anemia. The bone marrow of these patients usually demonstrates dyserythropoesis and variable dysmyelopoesis. It rarely progresses to leukemia or bone marrow failure $(1,2)$.

\subsection{Wiskott-Aldrich Syndrome (WAS)}

This disease is a rare condition with features of microthrombocytopenia, autoimmune problems, and eczema. The mode of inheritance is X-linked, and the defect of the WAS gene is located on Xp11.22. The features of this disease vary and can include microthrombocytopenia, eczema, and immune deficiency $(1,2)$.

WAS is most often diagnosed during the newborn period due to bleeding after circumcision or intracranial or gastrointestinal bleeding. Eczema may be present later in infancy. Infections and autoimmune disorders are other common presentations of this disease that may appear in later life. One common problem in these patients is the tendency to develop malignancies, which may arise after the age of 10 years.

Thrombocytopenia usually presents shortly after birth with a platelet count of about 5,000 -50,000. The size of platelets is small $(1.8 \mu \mathrm{M})$. In the bone marrow, there is a normal number of megakaryocytes, but they are unable to produce enough platelets, and their lifespan is shorter than normal. T-cell function is also abnormal. The IgM level is diminished, but the level of IgG immunoglobulin is normal (1-3).

\subsubsection{Treatment}

Severe bleeding should be managed with irradiated, HLA-matched platelets. Infection must be treated with proper antibiotics as well as other conventional and antifungal therapies through the intravenous route. Any autoimmune problem in these patients has to be carefully checked, and intravenous immunoglobulin (IVIG) should be administered monthly $(1,2,11-16)$.

\subsection{Case Presentation}

Case 1: This patient was the third child of nonconsanguineous parents. The patient was male and had been born at term via a normal vaginal delivery with a birth weight of 3620 gr and had a history of neonatal jaundice and phototherapy; the other two children were normal. At the age of 5 days, the patient was admitted to the hospital because of jaundice and had a platelet count of $27,000 / \mathrm{mm}^{3}$ without bleeding. At that time, he received IVIG and a platelet transfusion with no response, so he was given a second dose of IVIG. The next platelet count was $65,000 / \mathrm{mm}^{3}$. On the $23^{\text {rd }}$ day after birth, his CBC was as 
follows: WBCs: $12,500 / \mathrm{mm}^{3}$; RBCs: $3,160,000 / \mathrm{mm}^{3}$; MCV: $86 \mathrm{fl}$; Platelets: 27,000/$/ \mathrm{mm}^{3}$; and MPV: $5.9 \mu \mathrm{m}$. BMA was normocellular. After the BMA, prednisone $2 \mathrm{mg} / \mathrm{kg} /$ day was started. The patient's platelet count rose to $107,000 / \mathrm{mm}^{3}$, although he remained under observation.

After a routine vaccination at the age of two months, the platelet count again decreased, and treatment was repeated with corticosteroids and IVIG. The platelet count during the corticosteroid treatment was between 50,000 $-60,000 / \mathrm{mm}^{3}$. At the age of four months, he had two generalized, tonic-clonic seizures without fever. At this time, his platelet count was $44,000 / \mathrm{mm}^{3}$. Brain computed tomography (CT) and electroencephalogram (EEG) were normal. No bleeding was identified, and phenobarbital was started. A brain magnetic resonance imaging (MRI) scan showed benign external hydrocephalus. Follow-up was recommended with a pediatric neurologist. The patient's milestones of development continued to be normal. During this time, he was referred to hour hospital for further evaluation due to refractory thrombocytopenia and seizure disorder. Upon admission, his weight was $7.9 \mathrm{~kg}$, and had a mild to moderate eczematous rash on his face. Deeper investigation revealed that a maternal uncle of the patient suffered from severe eczema and severe thrombocytopenia and had died at age of 5 years due to bleeding.

Laboratory evaluation revealed the following: WBCs: 10,000/ $\mathrm{mm}^{3}$; neutrophils: 66\%; lymphocytes: $24 \%$; RBCs: 3.260,000/ $/ \mathrm{mm}^{3}$; Hb: $8.6 \mathrm{gr} / \mathrm{dL}$; MCV: $82 \mathrm{fl}$; platelets: 35,000/ $/ \mathrm{mm}^{3}$; MPV: $6 \mathrm{fl}$. Small platelets were seen on the PBS. Retic: 1\%; coombs direct and indirect: negative; PT: Negative; PTT: negative; virology tests (including EBV, CMV, hepatitis, and HIV): negative; collagen vascular tests biochemistry, thyroid function tests, urinalysis, and venous blood glucose: normal; BMA: normocellular with increased MKs; flow cytometry: normal; IgG:2,636 (180 - 800); IgM: 168 (20 - 100); IgA: 106 (10 - 131); IgE: 24 (up to 10). The patient had history of receiving IVIG on several occasions before admission to our hospital, so the results of immunoglobulin levels are not reliable. Chest X-ray (CXR) and abdominal ultrasound were normal.

During evaluation at our hospital, the patient had two episodes of seizure. EEG showed minimal abnormalities. Treatment with Liskantine was started. The findings in this child, including male sex, thrombocytopenia since the neonatal period, mild to moderate eczema, low MPV ( $5 \mathrm{fl})$, and a positive familial history, were compatible with WAS. Therefore, we conducted a DNA analysis for gene mutations (WASP Gene). We consulted a hematopoietic stem cell transplantation team, and this patient is now on the waiting list for (HSCT).

Case 2: A 2.7-year old boy who was the second child of non-consanguineous parents, was born at term via a
Cesarean section, and had exhibited normal growth and development was referred to our hospital due to persistent thrombocytopenia. He had a history of only one low platelet count during the neonatal period, when he was hospitalized for neonatal jaundice. After discharge, he was not followed-up for platelet count monitoring.

At the age of 6 months, the boy's parents noticed mild ecchymoses on the lower limbs and trunk of the patient. At 12 months of age, the patient visited a hematologistoncologist because of petechiae and ecchymoses. CBC revealed a severe thrombocytopenia. An evaluation that included a BMA, virology tests, and collagen vascular tests was carried out. The results of the BMA are not available, but the patient received IVIG and corticosteroids. He did not respond to treatment. During the course of the disease due to severe thrombocytopenia, which produced petechiae, ecchymoses, and nasal bleeding, the patient received cyclosporine and azathioprine in addition to prednisone and IVIG but showed no response.

The boy was once more referred to our hospital at two years of age for further evaluation. Upon physical examination, he had pallor, severe ecchymoses, and mild nasal bleeding. He weighed $10 \mathrm{~kg}$, and his milestones of development were normal. The results of his laboratory tests were as follows: WBCs: $4,200 / \mathrm{mm}^{3}$; RBCs $1,800,000 / \mathrm{mm}^{3}$; $\mathrm{Hb}: 6 \mathrm{gr} / \mathrm{dl}$; MCV: $98 \mathrm{fl}$; platelets: 13,000/$/ \mathrm{mm}^{3}$; retics: 2.2\%; coombs direct and indirect: negative; MPV: 9.9 fl; antiplatelet antibody tests: negative; PT: negative; PTT: negative; von Willebrand factor assay: negative; flow cytometry (CD42): normal; virology tests: normal; collagen vascular tests: normal; biochemistry: normal; Liver function: normal; kidney function and thyroid function tests: normal; parents' CBC: normal; CXR: normal; abdominal ultrasound: normal; first BMA and bone marrow biopsy (BMB): hypocellularity; second BMA: low MKs.

Because of pancytopenia on different CBCs, an evaluation for aplastic anemia was done. The patient's cytogenetic study was normal, and the karyotyping revealed 46XY; the chromosomal breakage (DEB) test was negative. The patient was also negative for the Wiskott-Aldrich syndrome (WASP) gene mutation, but the c-MPL gene was homozygous mutation-gene compatible with CAMT.

After this CAMT diagnosis, HLA typing was carried out in this patient's brother and parents, but they were not a match. While waiting for an HLA-compatible donor, he received supportive therapy, including irradiated platelets and irradiated, leukoreduced packed RBCs. Finally, the patient underwent HSCT from a matched, unrelated donor's stem cells at the age of 2.5 years. He had only a moderate episode of graft versus host disease, which improved following the administration of immunosuppressive agents. He is now in good condition and remains under our obser- 
vation.

\section{Results}

Although hereditary platelet disorders are individually rare, they are one of the most important causes of mucocutaneous bleeding. In patients with a family history that suggests a heritable condition or in persons with significant, lifelong bleeding histories, careful evaluation for the presence of hereditary platelet disorders is recommended. Early diagnosis and treatment significantly affects the prognosis of these groups of bleeding disorders.

\section{Conclusions}

Prompt recognition of the cause of thrombocytopenia is often crucial for the correct management of patients. Once considered a rarity, the inherited thrombocytopenia syndromes are increasingly being recognized as a spectrum of clinical disorders ranging from severe diseases in neonates to mild conditions that are only incidentally identified in adults. The heritable platelet disorders are a heterogeneous group; treatment options for bleeding can therefore be generalized. Stem cell transplantation is also an option for the most serious disorders.

\section{References}

1. Drachman JG. Inherited thrombocytopenia: when a low platelet count does not mean ITP. Blood. 2004;103(2):390-8. doi:10.1182/blood2003-05-1742. [PubMed: 14504084].

2. Bolton-Maggs PH, Chalmers EA, Collins PW, Harrison P, Kitchen S, Liesner RJ, et al. A review of inherited platelet disorders with guidelines for their management on behalf of the UKHCDO. Br J Haematol. 2006;135(5):603-33. doi: 10.1111/j.1365-2141.2006.06343.x. [PubMed: 17107346].

3. Cines DB, Bussel JB, McMillan RB, Zehnder JL. Congenital and acquired thrombocytopenia. 1. USA: ASH Education Program Book; 2004. pp. 390-406.
4. Stasi R. How to approach thrombocytopenia. 1. USA: ASH Education Program Book; 2012. pp. 191-7.

5. Al-Qahtani FS. Congenital amegakaryocytic thrombocytopenia: a brief review of the literature. Clin Med Insights Pathol. 2010;3:25-30. [PubMed: 21151552].

6. Althaus K, Greinacher A. MYH9-related platelet disorders. Semin Thromb Hemost. 2009;35(2):189-203. doi: 10.1055/s-0029-1220327. [PubMed: 19408192].

7. Balduini CL, Pecci A, Savoia A. Recent advances in the understanding and management of MYH9-related inherited thrombocytopenias. Br J Haematol. 2011;154(2):161-74. doi: 10.1111/j.1365-2141.2011.08716.x. [PubMed: 21542825].

8. Pecci A, Gresele P, Klersy C, Savoia A, Noris P, Fierro T, et al. Eltrombopag for the treatment of the inherited thrombocytopenia deriving from MYH9 mutations. Blood. 2010;116(26):5832-7. doi: 10.1182/blood2010-08-304725. [PubMed: 20844233].

9. Ghauri RI, Naveed M, Mannan J. Congenital amegakaryocytic thrombocytopenic purpura (CAMT). J Coll Physicians Surg Pak 2014;24(4):285-7. [PubMed: 24709246].

10. Ballmaier M, Germeshausen M, Schulze H, Cherkaoui K, Lang S, Gaudig A, et al. c-mpl mutations are the cause of congenital amegakaryocytic thrombocytopenia. Blood. 2001;97(1):139-46. [PubMed: 11133753].

11. Notarangelo LD, Miao $\mathrm{CH}$, Ochs HD. Wiskott-Aldrich syndrome. Curr Opin Hematol. 2008;15(1):30-6. doi: 10.1097/MOH.0b013e3282f30448. [PubMed: 18043243].

12. Jin Y, Mazza C, Christie JR, Giliani S, Fiorini M, Mella P, et al. Mutations of the Wiskott-Aldrich Syndrome Protein (WASP): hotspots, effect on transcription, and translation and phenotype/genotype correlation. Blood. 2004;104(13):4010-9. doi: 10.1182/blood-2003-05-1592. [PubMed: 15284122].

13. Ariga T. Wiskott-Aldrich syndrome; an x-linked primary immunodeficiency disease with unique and characteristic features. Allergol Int. 2012;61(2):183-9. doi: 10.2332/allergolint.11-RAI-0412. [PubMed: 22361515].

14. Syrigos KN, Makrilia N, Neidhart J, Moutsos M, Tsimpoukis S, Kiagia $\mathrm{M}$, et al. Prolonged survival after splenectomy in Wiskott-Aldrich syndrome: a case report. Ital J Pediatr. 2011;37:42. doi: 10.1186/1824-7288-3742. [PubMed: 21906397].

15. Leblebisatan G, Bay A, Mitsuiki N, Ohara O, Honma K, Imai K, et al. Wiskott-Aldrich syndrome mutation in two Turkish siblings with X-linked thrombocytopenia. Turk J Haematol. 2011;28(2):139-41. doi: 10.5152/tjh.2011.31. [PubMed: 27264129].

16. Lambert MP. What to do when you suspect an inherited platelet disorder?. Hematol Am Soc Hematol Educ Program. 2011;2011:377-83. 